\title{
Wavelength-Converter Saving Span Restoration in GMPLS Controlled WDM Optical Networks
}

Ruepp, Sarah Renée; Buron, Jakob Due; Andriolli, N.

\section{Published in:}

The Joint International Conference on Optical Internet and Next Generation Network, 2006. COIN-NGNCON 2006.

Link to article, DOI:

10.1109/COINNGNCON.2006.4454649

Publication date:

2006

Document Version

Publisher's PDF, also known as Version of record

Link back to DTU Orbit

Citation (APA):

Ruepp, S. R., Buron, J. D., \& Andriolli, N. (2006). Wavelength-Converter Saving Span Restoration in GMPLS Controlled WDM Optical Networks. In The Joint International Conference on Optical Internet and Next

Generation Network, 2006. COIN-NGNCON 2006. IEEE. https://doi.org/10.1109/COINNGNCON.2006.4454649

\section{General rights}

Copyright and moral rights for the publications made accessible in the public portal are retained by the authors and/or other copyright owners and it is a condition of accessing publications that users recognise and abide by the legal requirements associated with these rights.

- Users may download and print one copy of any publication from the public portal for the purpose of private study or research.

- You may not further distribute the material or use it for any profit-making activity or commercial gain

- You may freely distribute the URL identifying the publication in the public portal 


\title{
Wavelength-Converter Saving Span Restoration in GMPLS Controlled WDM Optical Networks
}

\author{
Sarah Ruepp ${ }^{1}$, Jakob Buron ${ }^{1}$, Nicola Andriolli ${ }^{2}$ \\ ( $\mathrm{COM} \cdot \mathrm{DTU}$, Technical University of Denmark, Oersteds Plads, Bygning 345v, $2800 \mathrm{Kgs}$. Lyngby, Denmark, \\ Tel: +45-4525-3627, Fax: +45-4593-6581, Email: \{sr, jbu\}@com.dtu.dk) \\ ( ${ }^{2}$ Scuola Superiore Sant'Anna di Studi Universitari e di Perfezionamento, Via Moruzzi 1, 56124 Pisa, Italy, \\ Tel: +39-050-5492170, Fax: +39-050-5492194, Email: nick@sssup.it)
}

\begin{abstract}
We present two label preference schemes to reduce wavelength-conversion during restoration path setup in GMPLS controlled optical networks exploiting span restoration. The amount of required wavelength-conversions can be reduced up to 34 percent.
\end{abstract}

\section{Introduction}

Span restoration is a well known method for achieving fast recovery of span failures in optical networks [1]. In span restoration, the affected connections are restored between the failure adjacent nodes, which means that the paths between the failure adjacent nodes and the source and destination nodes respectively (i.e. the stubs) remain unaffected. In order to restore a failed connection in an optical network, a control mechanism that can take care of finding a route and assigning a wavelength for the affected connection is required.

Generalized Multi-Protocol Label Switching (GMPLS) [2], which has evolved from the MPLS control plane, is a strong candidate for controlling next generation optical networks. In GMPLS, each label can represent a timeslot, wavelength or fiber. Wavelength Division Multiplexed (WDM) networks consist of combs of optical wavelengths; and with GMPLS control, each of these wavelengths gets a corresponding label assigned. At the different nodes (i.e. cross-connects), the traffic can be switched at wavelength granularity. In all-optical networks, a wavelength could traditionally only be used if it was available on the connection's entire route. But emerging technologies allow equipping network nodes with wavelength-converters, making it possible to change from one wavelength to another along the envisaged route. Usually, only a limited number of wavelength-converters is available in each node, because they are expensive and should therefore only be used if no continuous wavelength is available on the desired route.

The goal of network restoration is to restore as many failed connections as possible. Therefore, the use of wavelength-converters must be efficiently controlled both during the provisioning and the restoration phase in order to avoid the situation, where enough spare capacity actually is available on the remaining spans to restore the connection, but the lightpath cannot be converted to the desired wavelength due to the lack of available converters. To minimize the use of wavelength-converters in a GMPLS controlled network during Label Switched Path (LSP) setup, a signaling protocol extension called Suggested Vector (SV) has been proposed in [3]. The SV is used together with the label set, which is a standard protocol extension used by an upstream node to control the label selection of a downstream node. The SV collects information on the amount of wavelength-conversions the choice of a specific label requires to setup a connection. Based on these SV values, the destination node can then choose the label resulting in fewest wavelength-conversions along an envisaged route.

\section{Suggested Vector Restoration Schemes}

In this study, we investigate the performance of the SV principle for converter-saving span restoration in WDM optical networks. The failure of a single span interrupts all connections routed over that particular span. This results in many simultaneous restoration requests to 
recover the affected connections. To make a local detour around the failed span, both a route and a wavelength need to be found. The route is found by using the Dijkstra algorithm to identify the shortest path around the failure over spans with sufficient capacity available. The wavelength should be chosen such that the use of wavelength-converters is minimized. By using the SV principle, the different wavelengths can be given a priority depending on how many wavelength-conversions the choice of a particular wavelength imposes on the connection. Especially when many simultaneous connection requests need to be accommodated due to a span failure, it is important to limit the use of wavelength-converters, as leaving more wavelength-converts for other connections can increase the total restorability in the network.

We investigate two SV schemes, which differ on the point of stub-awareness. In the stub-unaware scheme, the detour path is signalled as a new regular connection, without considering that the connection stubs are already routed on specific wavelengths. Therefore, the upstream failure adjacent node, behaving as a regular connection source node, is indifferent of the label used on the previous hop by the failed connection. This means that all available labels are given the same SV value of 0 . At any intermediate node, if an outgoing label is also available on the previous hop, the SV will keep its value. If the label is not available on the previous hop, its SV is computed as the minimum SV value on the previous hop plus 1 , because a wavelength-conversion is required. At the downstream failure adjacent node only the received SV is considered when choosing the label. It is thereby indifferent to the downstream stub label. The principle is illustrated in Figure 1.

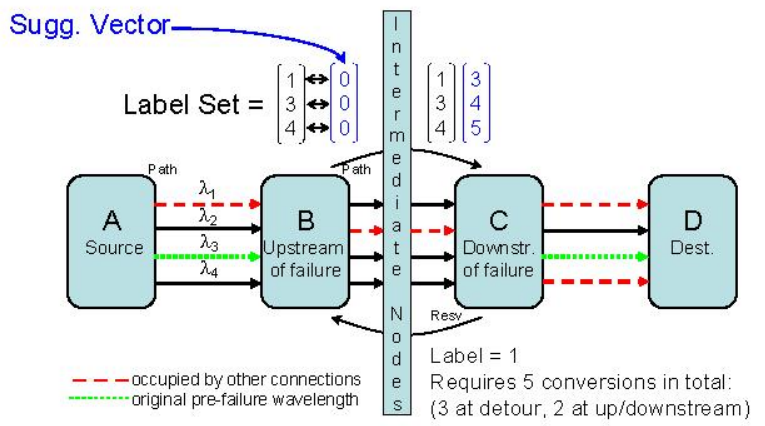

Figure 1: Stub-unaware SV label assignment
In addition, we propose a stub-aware scheme, where we take into account that failure adjacent nodes may require wavelength-conversion to match the detour path wavelength to the stub wavelength. The SV values now represent the total amount of required wavelength-conversions for the backup connection, including conversion to stub wavelengths. To reflect that, the upstream failure adjacent node modifies the SV in such a way, that the stub label is given a SV value of 0 , since its choice will require no conversion at the upstream failure adjacent node. Any other wavelengths will require a conversion and are therefore given a SV value of 1 . Intermediate nodes perform their SV calculations corresponding to the stub-unaware scheme. When the downstream failure adjacent node is reached, it examines its downstream stub label and modifies the received SV by adding 1 to all other labels, since they require a wavelength-conversion to the stub label. The label is chosen as the wavelength which requires fewest conversions at the upstream failure adjacent node, the intermediate detour nodes and the downstream failure adjacent node in total. The principle is illustrated in Figure 2, where green circles mark the stub-aware modifications.

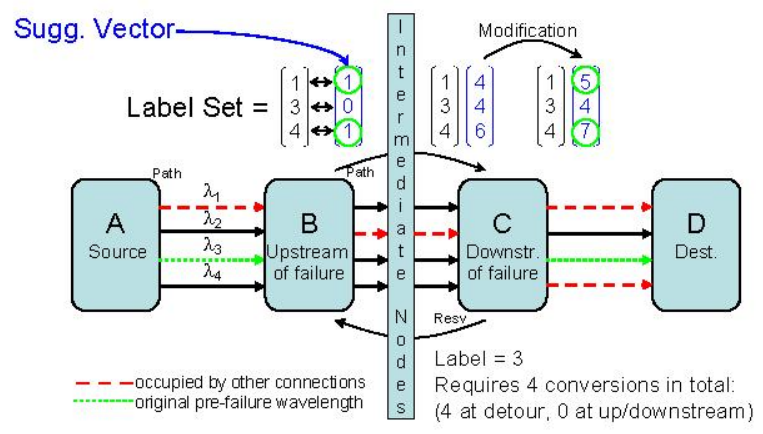

Figure 2: Stub-aware SV label assignment

\section{Simulation Study and Results}

In this study, we investigate the use of wavelength-converters for span restoration, and compare the following schemes' capabilities for converter saving: No preference (a label is chosen amongst the free wavelengths on the last hop only), SV without stub-awareness and SV with stub-awareness. First-fit tie-breaking policy is used for labels with equal SV values. The schemes are simulated in OPNET Modeler [4] and evaluated on the 
COST 266 Pan-European triangular topology network [5] illustrated in Figure 3. The network consists of 28 nodes and 61 spans, resulting in a nodal degree of 4.36. Each span has a capacity of 10 wavelengths, and each node has full wavelength-conversion capability.

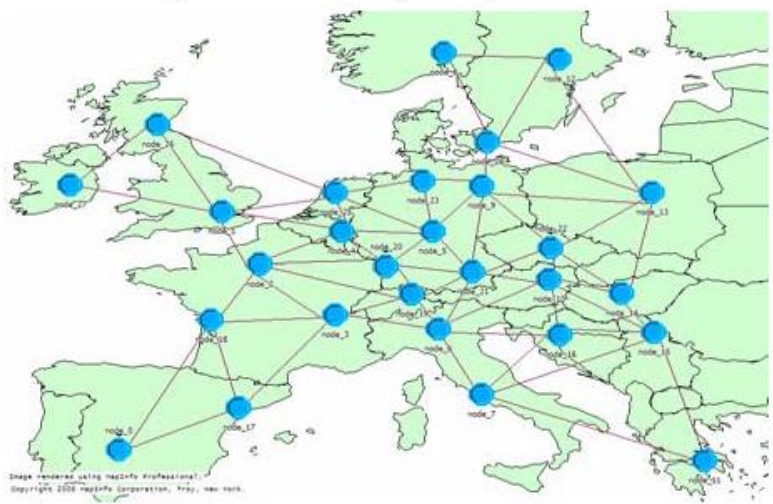

Figure 3: COST 266 Pan-European triangular topology network

We populate the network with unidirectional connections of unit wavelength size up to a desired average span load using the no preference scheme. The highest considered network load before failure is 0.6 in order to leave enough available capacity for restoration. When reaching the desired load, a span is failed and statistics for the converter count for the recovery paths are collected. After each failure simulation, the network is reversed to its pre-failure state before the next span failure is evaluated.

Figure 4 illustrates that the no preference scheme uses between 1.5 and 2.2 wavelength-converters per recovered connection averaged over all link failures. In comparison, the SV schemes use between 1.0 and 1.6 wavelength-converters per recovered connection, gaining a maximum of $34 \%$.

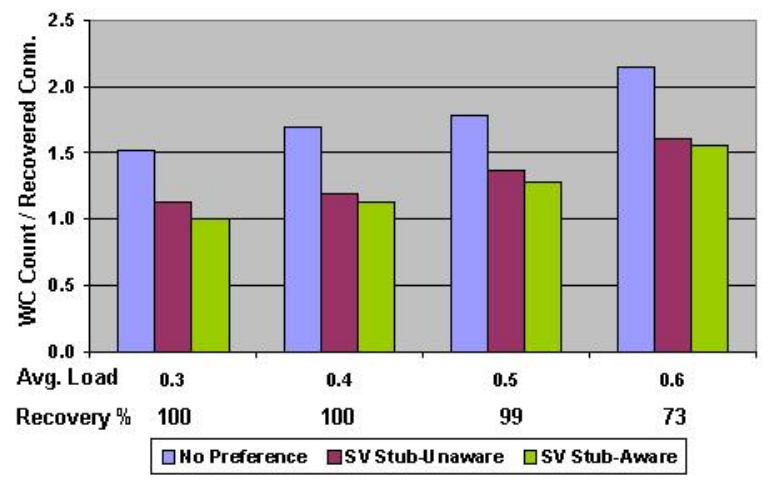

Figure 4: Wavelength converter count per recovered connection
Figure 5 shows that the stub-aware scheme is more efficient than the stub-unaware scheme by an $11 \%$ margin. The improvement due to stub-awareness diminishes to $3 \%$ at high network loads. This is because the label choice is limited due to the fact that only few free wavelengths are available at high loads.

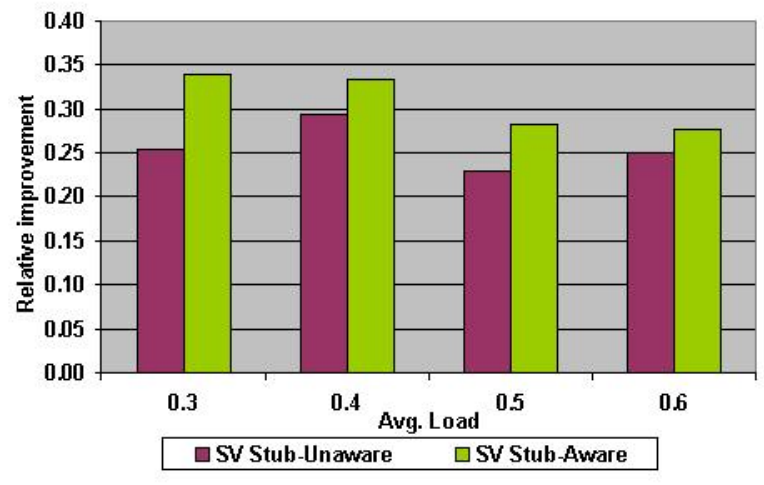

Figure 5: Relative improvement compared to no pref. scheme

\section{Conclusion}

We show that SV schemes applied to span restoration outperform the no preference scheme in terms of used wavelength-converters per recovered connection. The proposed stub-aware scheme significantly improves the SV principle for span restoration. Only failure adjacent nodes need to modify the SV, so the change is transparent to the intermediate nodes. Hence the additional control burden imposed by the stub-aware scheme is very limited.

\section{References}

1 Ramamurthy, S., Sahasrabuddhe, L., Mukherjee, B., Survivable WDM Mesh Networks. JT, Vol. 21, no. 4, Apr. 2003 pp. $870-883$

2 Mamie, E. (ed.), Generalized Multi-Protocol Label Switching (GMPLS) architecture, RFC 3945, October 2004

3 Andriolli, N., Buron, J., Ruepp, S., Cugini, F., Valcarenghi, L. and Castoldi, P., Signalling protocol extensions for convertersaving wavelength assignment in GMPLS optical networks accepted at HPSR 2006, Poznan, Poland, 2006.

4 OPNET Modeler, www.opnet.com

5 Inkret, R., Kuchar, A. and Mikac, B. (eds.), Extended Final Report of COST Action 266 - Advanced Infrastructure for Photonic Networks, 2003 